\title{
Accelerated Optical Topography Inspection Using Parameterized Model Order Reduction
}

\author{
Jung Hoon Lee*, Dimitry Vasilyev*, Anne Vithayathil ${ }^{\dagger}$, Luca Daniel*, and Jacob White* \\ * Electrical Engineering and Computer Science \\ Massachusetts Institute of Technology \\ 77 Massachusetts Avenue, Cambridge, MA 02139 \\ Email: junghoon@mit.edu \\ $\dagger$ Nanobiosym, Inc. \\ 200 Boston Avenue, Suite 4700 \\ Medford, MA 02055
}

\begin{abstract}
This paper describes an efficient method for solving an inverse optical scattering problem associated with the optical semiconductor process inspection. The method determines the geometric features of a fabricated structure, from spectroscopic ellipsometry measurements, by combining a parameterized low-order model with an optimization algorithm. We make improvements on the polynomial fittingbased parameterized moment matching technique to extract such model automatically. Since the resulting model is inexpensive to evaluate, the method shows large speedup without losing much accuracy: the examples show more than 1000 times speedup with less than $1 \%$ error in the final geometric parameter estimation.
\end{abstract}

\section{INTRODUCTION}

The technology for fabricating integrated circuit structures requires very finely tuned photolithographic-etching processes. Undercutting or over-rounding easily occurs, for instance, by erroneous acid concentrations and/or longer/shorter etch time. Consequently, accurate and online process monitoring techniques are vital to ensure correct fabrication parameters which produce consistent features.

A commonly used on-line monitoring device is spectroscopic ellipsometry: it illuminates an etched surface with light over a wide range of frequencies and measures the scattered field. Then, the actual shape of the scattering surface can be inferred from the scattered field. Because the scattered field does not directly give the shape information, inferring the shape of the scattering surface is a challenging inverse problem [1], [2], [3], [4] and must be solved rapidly as the inspection is performed on-line. Table lookup over a database of pre-computed scattered field and scatterer relations has been employed [5], but large tables are needed to achieve the desired accuracy.

In this paper, we reconstruct the shape of the scattering surface using a parameterized low-order model: the model simulates the measurement process, and the difference between the measured and the simulated scattered fields is minimized. If the model is a function of parameters describing the geometric features of the scattering surface and is accurate enough, the difference-minimizing parameters are close to the true ones.

The proposed method consists of four major steps: (a) an integral formulation describing the scattering; (b) a boundary element method for solving the integral equations; (c) a parameterized model order reduction algorithm to accelerate the model evaluation; and (d) an optimization algorithm to determine the geometric parameters by matching the model output to the measurement data. The steps from (a) to (c) create a parameterized low-order model directly from the Maxwell's equations, so that it is practically as accurate as a field solver but, at the same time, is inexpensive enough for repeated evaluations. Then, the model is coupled with a suitable optimization algorithm to deduce the unknown parameters in step (d).

Our main contributions are modifying the parameterized moment matching (PMM) techniques for oscillating functions and parameter dependent inputs; applying the polynomial fitting-based PMM technique in [6] to the optical scattering analysis, creating a parameterized loworder model; and combining the model with an optimization algorithm to perform a fast inverse optical scattering analysis.

\section{BACKGROUND}

\section{A. Integral Formulation and Boundary Element Method}

The scattering of an electromagnetic wave from an object can be modeled using the PMCHW formulation [7], [8], [9], [10]. The resulting coupled integral equations have tangential components of the surface electric and magnetic fields as unknowns, denoted $\mathbf{J}$ and $\mathbf{M}$ respectively; we can also interpret them as the equivalent surface electric and magnetic currents.

Then, a surface discretization can be introduced to convert the coupled integral equations into a system of algebraic equations: the surface is discretized into triangular panels, and $\mathbf{J}$ and $\mathbf{M}$ are approximated using weighted combinations of RWG basis functions [11]. Once the approximate $\mathbf{J}$ and $\mathbf{M}$ are computed, they can be used to determine the scattered field at any point in space. 
Suppose a set of parameters $\mathbf{p}=\left(p_{1}, p_{2}, \cdots, p_{k}\right)$ characterizes the geometric features of a scattering surface; in addition, $\mathbf{p}$ may also include the wavelength of the incident field. Then, for a given $\mathbf{p}$, the overall problem after discretization is

$$
\mathbf{A}(\mathbf{p}) \mathbf{x}(\mathbf{p})=\mathbf{b}(\mathbf{p}), \quad \mathbf{y}(\mathbf{p})=\mathbf{C}(\mathbf{p}) \mathbf{x}(\mathbf{p})
$$

where $\mathbf{A}(\mathbf{p})$ and $\mathbf{C}(\mathbf{p})$ are the discretized integral operators, $\mathbf{b}(\mathbf{p})$ is the vector of integrals of the incident field, half of the entries in $\mathbf{x}(\mathbf{p})$ represent the discretized $\mathbf{J}$, and the other half represent the discretized M [10]. The variable $\mathbf{y}(\mathbf{p})$ is the approximated scattered electric field at a given measurement point, and $\mathbf{C}(\mathbf{p})$ relates the surface electric and magnetic currents to the measured electric field. The entries of $\mathbf{A}(\mathbf{p}), \mathbf{b}(\mathbf{p})$, and $\mathbf{C}(\mathbf{p})$ are functions of $\mathbf{p}$, since the Green's function in the integral operator and the discretization of the surface change as functions of $\mathbf{p}$.

For a complicated inspection geometry, the number of unknowns, $N$, can exceed several thousand. This large number of unknowns implies that (1) is costly to solve.

\section{B. Model Order Reduction}

In order to reduce the cost of solving (1) to compute the scattered field $\mathbf{y}(\mathbf{p})$ due to a set of parameters $\mathbf{p}$, we use a technique referred to as parameterized model order reduction. In this section, we describe a parameterized model order reduction technique called the parameterized moment matching (PMM) method.

1) PMM: Consider an $N$-dimensional system of equations parameterized affinely with respect to $\mathbf{p}=$ $\left(p_{1}, p_{2}, \cdots, p_{k}\right)$ as

$$
\left(\mathbf{A}_{0}+p_{1} \mathbf{A}_{1}+\cdots+p_{k} \mathbf{A}_{k}\right) \mathbf{x}(\mathbf{p})=\mathbf{b},
$$

and we are interested in the response $\mathbf{y}(\mathbf{p})$ from

$$
\mathbf{y}(\mathbf{p})=\mathbf{C}(\mathbf{p}) \mathbf{x}(\mathbf{p})
$$

Using the projection strategy in [12] with a projection matrix $\mathbf{V} \in \mathcal{R}^{N \times n}$, (2) and (3) can be reduced to

$$
\begin{aligned}
\left(\hat{\mathbf{A}}_{0}+p_{1} \hat{\mathbf{A}}_{1}+\cdots+p_{k} \hat{\mathbf{A}}_{k}\right) \hat{\mathbf{x}}(\mathbf{p}) & =\hat{\mathbf{b}} \\
\hat{\mathbf{y}}(\mathbf{p}) & =\hat{\mathbf{C}}(\mathbf{p}) \hat{\mathbf{x}}(\mathbf{p})
\end{aligned}
$$

where $\hat{\mathbf{A}}_{i}=\mathbf{V}^{T} \mathbf{A}_{i} \mathbf{V}, \hat{\mathbf{b}}=\mathbf{V}^{T} \mathbf{b}$, and $\hat{\mathbf{C}}(\mathbf{p})=\mathbf{C}(\mathbf{p}) \mathbf{V}$. The reduced system in (4) is much lower in order than the original system if $n \ll N$.

In order for (4) to maintain fidelity, the projection matrix $\mathbf{V}$ must be chosen carefully; one approach shown in [13] chooses $\mathbf{V}$ so that the first few Taylor expansion coefficients of $\hat{\mathbf{y}}(\mathbf{p})$ in (4) match those of $\mathbf{y}(\mathbf{p})$ in (3).

2) Polynomial Fitting-Based PMM: In practice, the matrix $\mathbf{A}(\mathbf{p})$ as a function of $\mathbf{p}$ is not known explicitly, and it is also unlikely to be an affine function.

The polynomial fitting-based PMM in [6] samples the matrices at different points in the parameter space, sometimes referred to as snapshots, and fits the entries with polynomials. For a given matrix $\mathbf{A}(\mathbf{p})$, the polynomial fit $\tilde{\mathbf{A}}(\mathbf{p})$ is

$$
\tilde{\mathbf{A}}(\mathbf{p})=\tilde{\mathbf{A}}_{0}+\sum_{j} p_{j} \tilde{\mathbf{A}}_{j}+\sum_{j, k} p_{j} p_{k} \tilde{\mathbf{A}}_{j, k}+\cdots
$$

Unfortunately, (5) is not an affine function of $p_{i}$ 's. By redefining the parameters as

$$
p_{i}^{\prime}=\left\{\begin{array}{cc}
p_{j}, & j=1, \cdots, k \\
p_{j} p_{k}, & j=1, \cdots, k, k=1, \cdots, k \\
\vdots &
\end{array}\right.
$$

the resulting $\tilde{\mathbf{A}}\left(\mathbf{p}^{\prime}\right)$ is an affine function of a new set of parameters $\mathbf{p}^{\prime}=\left(p_{1}^{\prime}, p_{2}^{\prime}, \cdots\right)$, and the PMM in subsubsection II-B.1 can be applied. With a slight abuse of the notation in the rest of the paper, we use $\mathbf{p}$ and $p_{i}$ instead of $\mathbf{p}^{\prime}$ and $p_{i}^{\prime}$, respectively.

\section{IMPROVEMENTS IN INTERPOLATION-BASED PMM}

As explained in subsection II-A, the optical scattering problem is a function of some geometric parameters and possibly the wavelength, denoted as p. In this section, we investigate the additional issues related to constructing an affinely parameterized model $\tilde{\mathbf{A}}(\mathbf{p}), \tilde{\mathbf{b}}(\mathbf{p})$, and $\tilde{\mathbf{C}}(\mathbf{p})$ by fitting the snapshots of $\mathbf{A}(\mathbf{p}), \mathbf{b}(\mathbf{p})$, and $\mathbf{C}(\mathbf{p})$ with polynomials.

\section{A. Fitting in Frequency}

For the examples that we are considering, the size of the object is of the order of a wavelengths, but the measurement point is usually a number of wavelengths away. In this setting, the entries of $\mathbf{C}(\mathbf{p})$ are strong nonlinear functions of the wavelength. This is because the Green's function for the integral equation,

$$
G_{V}\left(\vec{r}-\vec{r}^{\prime}\right)=\frac{e^{-j \omega \sqrt{\varepsilon_{\varepsilon^{\prime}} \mu_{v}}}|| \vec{r}-\vec{r}^{\prime} \|}{4 \pi|| \vec{r}-\vec{r}^{\prime} \|},
$$

oscillates for large $\left\|\vec{r}-\vec{r}^{\prime}\right\|$, even with a small change in $\omega$.

To improve the accuracy of the polynomial fit, a nominal distance is factored out. Assume that there is a cluster of panels all within a range smaller than the wavelength $\lambda$, and let the center of the cluster be $\vec{r}_{0}$. By factoring out $\left\|\vec{r}-\vec{r}_{0}\right\|, G_{v}(\cdot)$ is

$$
\begin{aligned}
G_{v}\left(\vec{r}-\vec{r}^{\prime}\right) & =e^{-j \omega \sqrt{\varepsilon_{v} \mu_{v}}|| \vec{r}-\vec{r}_{0} \|} \cdot \frac{e^{-j \omega \sqrt{\varepsilon_{v} \mu_{v}}\left(\left|\vec{r}-\vec{r}^{\prime}\right||-| \mid \vec{r}-\vec{r}_{0} \|\right)}}{4 \pi\left\|\vec{r}-\vec{r}^{\prime}\right\|} \\
& =e^{-j \omega \sqrt{\varepsilon_{v} \mu_{v}}\left\|\vec{r}-\vec{r}_{0}\right\|} G_{v}^{\prime}\left(\vec{r}-\vec{r}^{\prime}\right)
\end{aligned}
$$

and $\left(\left\|\vec{r}-\vec{r}^{\prime}\right\|-\left\|\vec{r}-\vec{r}_{0}\right\|\right)$ in $G_{v}^{\prime}(\cdot)$ remains small. Hence, $G_{v}^{\prime}(\cdot)$ can be easily fitted with a polynomial. 


\section{B. De-parameterization of $\mathbf{b}(\mathbf{p})$}

For the scattering problem, $\mathbf{b}(\mathbf{p})$ is a function of $\mathbf{p}$ due to geometric variations or the change in the wavelength. Even after $\mathbf{b}(\mathbf{p})$ is fitted with

$$
\tilde{\mathbf{b}}(\mathbf{p})=\tilde{\mathbf{b}}_{0}+p_{1} \tilde{\mathbf{b}}_{1}+\cdots+p_{k} \tilde{\mathbf{b}}_{k},
$$

using the method in subsubsection II-B.2, we cannot apply PMM directly to find the projection matrix $\mathbf{V}$.

We modify the system $\tilde{\mathbf{A}}(\mathbf{p}), \tilde{\mathbf{b}}(\mathbf{p})$, and $\tilde{\mathbf{C}}(\mathbf{p})$ with affine dependency on the parameters to have constant right-hand side vector $\tilde{\mathbf{b}}^{\prime}$. Since the method is related to the PMM method in [13], and we are limited by space in this paper, the details will be given in a following Transaction paper. The system after the modification is

$$
\tilde{\mathbf{A}}^{\prime}(\mathbf{p}) \tilde{\mathbf{x}}^{\prime}(\mathbf{p})=\tilde{\mathbf{b}}^{\prime}, \quad \tilde{\mathbf{y}}^{\prime}(\mathbf{p})=\tilde{\mathbf{C}}^{\prime}(\mathbf{p}) \tilde{\mathbf{x}}^{\prime}(\mathbf{p}),
$$

and the PMM can be readily applied to find $\mathbf{V}$.

\section{InVERSE SCATtering Problem}

In order to estimate the geometric features of the scattering surface, we want to determine $\mathbf{p}$ such that $\mathbf{y}(\mathbf{p})$ in (1) matches the measured field $\mathbf{y}_{m}$ as closely as possible. This problem is stated as a least-squares problem [14]

$$
\min _{\mathbf{p} \in X}\|\mathbf{f}(\mathbf{p})\|^{2}
$$

where $\mathbf{f}(\mathbf{p})=\mathbf{y}(\mathbf{p})-\mathbf{y}_{m}$ and $X$ is the range of all admissible parameter values. In practice, the real and imaginary parts of $\mathbf{y}(\mathbf{p})$ are minimized separately to recover real parameters from the optimization problem.

A well-known algorithm for solving nonlinear leastsquares problems is the Levenberg-Marquardt algorithm, which is a trust-region variant of the Gauss-Newton algorithm [15]. To use this algorithm, we have to compute $\mathbf{f}(\mathbf{p})$, and its Jacobian matrix $\mathbf{F}_{i, j}=\frac{\partial f_{i}(\mathbf{p})}{\partial p_{j}}$ at each step. Unfortunately, solving for $\mathbf{f}(\mathbf{p})$ and $\mathbf{F}$ takes most of the computation time.

Using an affinely parameterized low-order approximation of (1),

$$
\begin{aligned}
& \left(\hat{\mathbf{A}}_{0}+p_{1} \hat{\mathbf{A}}_{1}+\cdots+p_{k} \hat{\mathbf{A}}_{k}\right) \hat{\mathbf{x}}(\mathbf{p})=\hat{\mathbf{b}} \\
& \hat{\mathbf{y}}(\mathbf{p})=\left(\hat{\mathbf{C}}_{0}+p_{1} \hat{\mathbf{C}}_{1}+\cdots+p_{k} \hat{\mathbf{C}}_{k}\right) \hat{\mathbf{x}}(\mathbf{p}),
\end{aligned}
$$

generated by the polynomial fitting-based PMM and the techniques in section III offers two main advantages. First, the entries of $\mathbf{F}$ are products of $\hat{\mathbf{A}}_{i}, \hat{\mathbf{C}}_{i}$, and $\hat{\mathbf{b}}$, which are straightforward to compute. Second, solving for both $\mathbf{f}(\mathbf{p})$ and $\mathbf{F}$ requires inverting $\hat{\mathbf{A}}(\mathbf{p})$, which is the small matrix associated with the reduced model. Note that applying the Levenberg-Marquardt to (1) directly would be substantially more expensive.

\section{Summary OF Algorithm}

- Pre-computing the model:

1) Compute the discretized integral operators $\mathbf{A}(\mathbf{p})$ and $\mathbf{C}(\mathbf{p})$ as well as the integrals of the incident field $\mathbf{b}(\mathbf{p})$ in (1) for a number of different $\mathbf{p}$ vectors in the parameter space (subsection IIA).

2) Fit the entries of $\mathbf{A}(\mathbf{p})$ 's, $\mathbf{b}(\mathbf{p})$ 's, and $\mathbf{C}(\mathbf{p})$ 's to get $\tilde{\mathbf{A}}(\mathbf{p}), \tilde{\mathbf{b}}(\mathbf{p})$, and $\tilde{\mathbf{C}}(\mathbf{p})$ as in (5) (subsubsection II-B.2 and subsection III-A).

3) De-parameterize $\tilde{\mathbf{b}}(\mathbf{p})$ to get $\tilde{\mathbf{A}}^{\prime}(\mathbf{p}), \tilde{\mathbf{b}}^{\prime}$, and $\tilde{\mathbf{C}}^{\prime}(\mathbf{p})$ (subsection III-B).

4) Compute the projection matrix $\mathbf{V}$ as in [13] for the de-parameterized system, and form the reduced model $\hat{\tilde{\mathbf{A}}}^{\prime}(\mathbf{p}), \hat{\tilde{\mathbf{b}}}^{\prime}$, and $\hat{\widetilde{\mathbf{C}}}^{\prime}(\mathbf{p})$ (subsubsection II-B.1).

- Estimating the geometric parameters:

1) Measure the field $\mathbf{y}_{m}$.

2) Solve (11) iteratively (section IV) by evaluating the objective function and its Jacobian matrix associated with the reduced model.

\section{EXAMPLES}

\section{A. Case One: a Sphere}

The scatterer is a ball of silicon dioxide $(\varepsilon=11.7, \mu=1$, and $\sigma=0)$ centered at the origin. The test conditions are

- The radius of the sphere is $r \in[95 \mathrm{~nm}, 105 \mathrm{~nm}]$ with the nominal value of $r_{0}=100 \mathrm{~nm}$.

- The incident field is a plane wave with $\lambda \in$ [190nm,210nm] with the nominal value of $\lambda_{0}=$ $200 \mathrm{~nm}$, coming from $\theta=\pi / 3$, polarized in the $\hat{x}$ direction, and the phase being zero at the origin.

- The scattered field is measured at distance $20.013 \lambda_{0}$ away from center in the $(1,1,1)$ direction.

The values for $\lambda / \lambda_{0}$ and $r / r_{0}$ are sampled at 0.95 , $0.99,1.01$, and 1.05 ; and they are fitted with a third order polynomial. Then, moments are matched at $\left(\lambda / \lambda_{0}, r / r_{0}\right)=$ $(1,1),(0.95,0.95)$ up to the first order. The reduced model is of size 30-by-30 compared to the original model of size 576-by-576, and it is parameterized in both the wavelength and the radius. As a result, our approach gives about a factor of 8000 times speedup in each model evaluation.

The exact values of the scattered field are evaluated at three different wavelengths to form $\mathbf{y}_{m}$. Fig. 1 shows $\|\mathbf{f}(\mathbf{p})\|$ versus the radius, and the optimizer has converged to a minimum of $97.3 \mathrm{~nm}$, identifying the true value $97 \mathrm{~nm}$ with an error of $0.3 \%$.

\section{B. Case Two: a Pillar}

The pillar shown in Fig. 2 is a simplification of a wall of silicon dioxide that is formed after each etch. It has width $w$ in both the $\hat{x}$ and the $\hat{y}$-directions and height $h$ in the $\hat{z}$-direction. The test conditions are 


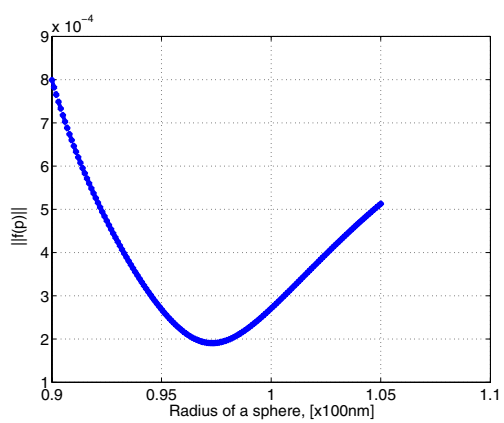

Fig. 1. Plot of the objective function in (11) evaluated with a parameterized reduced model for the sphere example.

- The width and the height are $w \in[22 \mathrm{~nm}, 28 \mathrm{~nm}]$ and $h \in[45 \mathrm{~nm}, 55 \mathrm{~nm}]$ with the nominal values of $w_{0}=$ $25 \mathrm{~nm}$ and $h_{0}=50 \mathrm{~nm}$, respectively.

- The same incident field and observation point are used as in the sphere case.

The values for $w / w_{0}$ and $h / h_{0}$ are sampled at $0.85,0.95$, 1.05 , and 1.15; and they are fitted with a third order polynomial. Then, moments are matched at $\left(w / w_{0}, h / h_{0}\right)=$ $(1,1)$ up to the second order. Note that the wavelength is not a parameter, and three separate models are built for different wavelengths. Each original model is of size 540by-540 and each reduced model is of size 50-by-50, which gives about a factor of 1000 times speedup.

Again, the exact values of the scattered field are evaluated to form $\mathbf{y}_{m}$. Fig. 2 shows $\|\mathbf{f}(\mathbf{p})\|$ versus the width, and the optimizer has converged to $104.9 \mathrm{~nm}$, identifying the true value of $105 \mathrm{~nm}$ with $0.1 \%$ error.
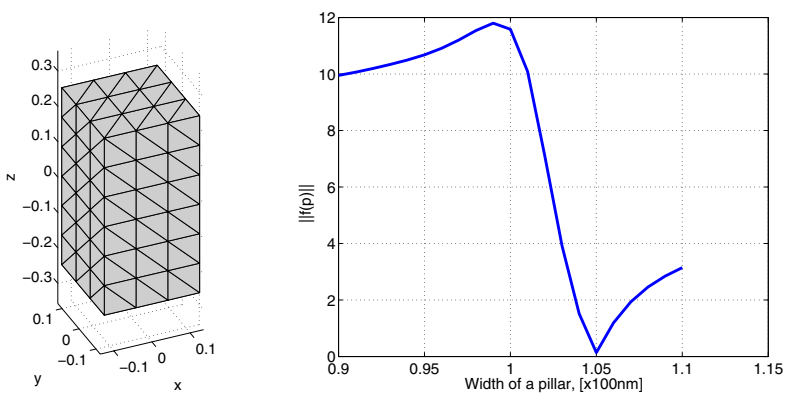

Fig. 2. Plot of the objective function in (11) with the parameterized reduced model for the pillar example.

\section{CONCLUSION}

In this paper, we described an approach to efficiently solve an inverse optical scattering problem associated with the optical semiconductor process inspection. The method combines the PMCHW formulation, boundary element method, and parameterized moment matching to form a parameterized low-order model; and the LevenbergMarquardt algorithm is used to minimize the least-squares difference between the measured and computed scattered fields. Coupling an optimization algorithm with a parameterized low-order model significantly reduces the computational cost associated with repeated model evaluation.

The approach was demonstrated on a silicon dioxide sphere and a pillar to estimate their radius and width, respectively. We achieved factors of 1000 and 8000 reduction in computation, while successfully identifying the parameters of interest with errors of $0.3 \%$ and $0.1 \%$.

\section{ACKNOWLEDGMENT}

The authors would like to acknowledge support from the MARCO designated interconnect focus centers, the Semiconductor Research Corporation, the Singapore-MIT Alliance, and the National Science Foundation.

\section{REFERENCES}

[1] P. Schiavone, V. Farys, and A Michallet. Analysis of feature shape variation using scatterometry. In Proceedings of SPIE, volume 4691-19, 2002.

[2] C. J. Raymond, M. R. Murnane, S. L. Prins, S. Sohail, H. Naqvi, J. R. McNeil, and J. W. Hosch. Multiparameter grating metrology using optical scatterometry. Journal of Vacuum Science \& Technology $B, 15(2): 361-368,1997$.

[3] X. Niu, N. Jakatdar, J. Bao, and C. J. Spanos. Specular spectroscopic scatterometry. IEEE Transactions on Semiconductor Manufacturing, 14(2):97-111, 2001.

[4] J. A. Allgair, D. C. Benoit, M. Drew, R. R. Hershey, L. C. Litt, P. P. Herrera, U. K. Whitney, M. Guevremont, A. Levy, and S. Lakkapragada. Implementation of spectroscopic critical dimension (SCD) for gate CD control and stepper characterization. Proceedings of SPIE, Metrology, Inspection, and Process Control for Microlithography XV, 4344:462-471, 2001.

[5] Q. Wu, D. Djajaputra, M. Lauterbach, Y. Wu, and R. Mohan. A fast dose calculation method based on table lookup for IMRT optimization. Physics in Medicine and Biology, 48(12):N159N166, 2003.

[6] L. Daniel and J. White. Automatic generation of geometrically parameterized reduced order models for integrated spiral rf-inductors. In Proceedings of BMAS, San Jose, California, 2003.

[7] R. F. Harrington. Boundary integral formulation for homogeneous material bodies. Journal of Electromagnetic Waves and Applications, pages $1-15,1989$.

[8] A. J. Poggio and E. K. Miller. Integral equation solutions of three-dimensional scattering problems. In Computer Techniques for Electromagnetics, pages 159-264. Hemisphere, 1987.

[9] L. N. Medgyesi-Mitschang, J. M. Putnam, and M. B. Gedera. Generalized method of moments for three-dimensional penetrable scatterers. Journal of the Optical Society of America A, pages 13831398, 1994.

[10] B. H. Jung, T. K. Sarkar, and Y. S. Chung. A survey of various frequency domain integral equations for the analysis of scattering from three-dimensional dielectric objects. Progress in Electromagnetics Research, pages 193-246, 2002.

[11] S. M. Rao, D. R. Wilton, and A. W. Glisson. Electromagnetic scattering by surfaces of arbitrary shape. IEEE Transactions on Antennas and Propagation, pages 409-418, 1982.

[12] E. Grimme. Krylov Projection Methods for Model Reduction. PhD thesis, Coordinated-Science Laboratory, University of Illinois at Urbana-Champaign, Urbana-Champaign, IL, 1997.

[13] L. Daniel, O. C. Siong, L. S. Chay, K. H. Lee, and J. White. A multiparameter moment matching model reduction approach for generating geometrically parameterized interconnect performance models. IEEE Transactions on Computer Aided Design, 23(5):678693, May 2004.

[14] C. R. Vogel. Computational Methods for Inverse Problems. SIAM, 2002.

[15] J. E. Dennis Jr. Non-linear least squares and equations. In The State of the Art in Numerical Analysis, pages 267-312. Academic Press, 1977. 